\title{
Generation of Stable Picosecond Pulses from an Electronically Wavelength-Tuned Laser for Material Processing
}

\author{
Yasuhiro Maeda ${ }^{1,2}$, Masaki Yumoto ${ }^{2}$, Norihito Saito ${ }^{2}$, Takayo Ogawa ${ }^{2}$, Masafumi Yamashita ${ }^{3}$, Satoshi Wada ${ }^{2}$ \\ ${ }^{1}$ Department of Physics, Graduate School of Science, Tokyo University of Science, 1-3 Kagurazaka, \\ Shinjuku, Tokyo 162-8601, Japan \\ ${ }^{2}$ Optical Green Technology Research Unit, RIKEN 2-1 Hirosawa, Wako, Saitama 351-0198, Japan \\ E-mail: yasumaeda@riken.jp \\ ${ }^{3}$ Department of Physics, Faculty of Science and Technology, Tokyo University of Science, 2641 Ya- \\ mazaki, Noda, Chiba 287-8510, Japan
}

We generated stable mode-locked picosecond pulses from an electronically wavelength-tuned laser. An acousto-optic tunable filter was placed in the cavity of a $\mathrm{Ti}_{\mathrm{Al}} \mathrm{Al}_{2} \mathrm{O}_{3}$ laser. The laser maintained picosecond operation even when the oscillation wavelength was switched at 1-ms intervals. We experimentally observed the behavior of the pulse train at the wavelength switching points.

DOI: $10.2961 / \mathrm{j} 1 \mathrm{mn} .2011 .01 .0005$

Keywords: picosecond pulse, mode locking, tunable laser, $\mathrm{TiAl}_{2} \mathrm{O}_{3}$ laser, $\mathrm{AOTF}$

\section{Introduction}

Mode-locked picosecond pulsed lasers are attracting considerable interest for laser processing [1-6] because they can perform nonthermal processing. The light sources for this application employ a master-oscillator power amplifier (MOPA) [7]. Picosecond pulses are generated from the laser oscillator by using a solid-state or fiber lasers are used with a semiconductor saturable absorber mirror (SESAM) [8] for mode locking. This is a passive modelocking technique. The advantages of this method are its simple configuration and stability against changes in the temperature and the cavity length. However, the damage and variations in the optical characteristic of the SESAM are problems for long-term operation. Active mode-locking can be performed with an acousto-optic (AO) modulator. However, this method is difficult to use in practical applications since it requires precisely matching the cavity length to the rf signal supplied to the AO. Therefore, more stable and practical mode-locking techniques for generating picosecond pulses are strongly required to develop more advanced laser processing systems. In previous studies, we developed electronically tuned nanosecond and picosecond pulsed Ti: $\mathrm{Al}_{2} \mathrm{O}_{3}$ lasers [9-11]. The electronically tuned picosecond pulsed laser has an acousto-optic tunable filter (AOTF) as a tuning element in its cavity [12-16]. It can generate stable picosecond pulses. Its oscillation wavelength can be switched at 1-ms intervals while maintaining a mode-locked 10-ps pulse train in the tuning range 739 to $868 \mathrm{~nm}$ without any dispersion compensation [11].

In the present study, we use an electronically wavelength-tuned $\mathrm{Ti}: \mathrm{Al}_{2} \mathrm{O}_{3}$ laser to demonstrate that mode licking with an AOTF is a practical method for generating picosecond pulses from a laser oscillator.

\section{Experiments}

Figures 1(a) and (b) respectively show the configuration and tuning curve of the electronically tuned picosecond pulsed $\mathrm{Ti}: \mathrm{Al}_{2} \mathrm{O}_{3}$ laser. A laser-diode-pumped frequency-doubled cw Nd: $\mathrm{YVO}_{4}$ laser was used to pump the

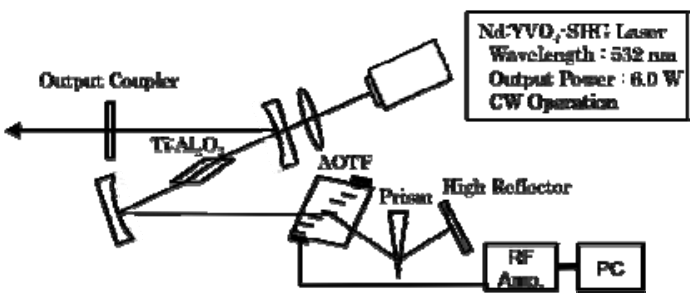

(a)

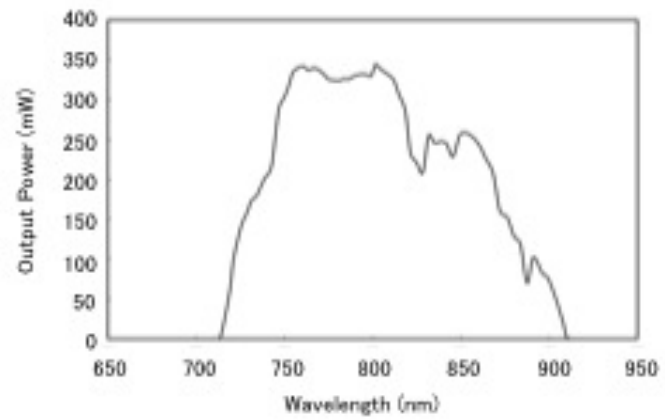

(b)

Fig. 1 Configuration (a) and tuning curve (b) of high-speed wavelength switching picosecond pulsed laser.

$\mathrm{TiAl}_{2} \mathrm{O}_{3}$ crystal at a pumping power of $6.0 \mathrm{~W}$. The cavity of the electronically tuned picosecond pulsed $\mathrm{Ti}: \mathrm{Al}_{2} \mathrm{O}_{3}$ laser consisted of a high reflector, an output coupler, and two concave mirrors arranged in a Z-fold type laser cavity. The cavity length was about $60 \mathrm{~cm}$. The wavelengths of the high reflector with a reflectance of $99.9 \%$ and the output coupler with a reflectance of $97 \%$ ranged from 680 to 1100 $\mathrm{nm}$. The concave mirrors had radii of curvature of $75 \mathrm{~mm}$. The Ti: $\mathrm{Al}_{2} \mathrm{O}_{3}$ crystal was set between the concave mirrors. An AOTF and a collection prism were inserted on one side of the cavity arms. An rf signal was generated by an rf amplifier, and its frequency and power were controlled by a personal computer. An acoustic wave was propagated in the AOTF by feeding the rf signal into the AOTF, and an incident beam was diffracted by the acoustic wave in the AOTF. 
The diffracted wavelength was controlled by varying the $\mathrm{rf}$ signal applied to the AOTF.

\section{Results and discussion}

The tuning range was 709 to $910 \mathrm{~nm}$, of which the picosecond pulsed oscillation region was from 739 to $868 \mathrm{~nm}$ [11]. The maximum output power was $344 \mathrm{~mW}$ at 801.5 $\mathrm{nm}$. The pulse duration was $10 \mathrm{ps}$ and repetition rate was $250 \mathrm{MHz}$. Figure 2 shows the autocorrelation pulse shape at $780 \mathrm{~nm}$. The pulse was assumed to be a $\operatorname{sech}^{2}$-shaped pulse. At this wavelength, the full width at half maximum

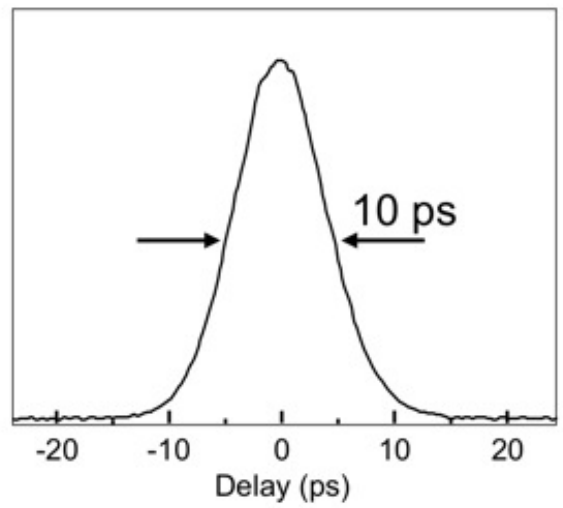

Fig. 2 Autocorrelation pulse shape obtained from oscillation of the electronically tuned picosecond pulsed Ti: $\mathrm{Al}_{2} \mathrm{O}_{3}$ laser with an autocorrelator (Model 409, Spectra Physics, Inc.). It has a pulse duration of $10 \mathrm{ps}$ assuming it is a $\operatorname{sech}^{2}$ shape pulse.

(FWHM) of the laser pulses was about 10 ps. The laser generated approximately 10-ps pulses in the wavelength tuning range. The Fourier-transform-limited pulse duration was determined to be $3.2 \mathrm{ps}$ from the product of the pulsewidth and linewidth for a $\operatorname{sech}^{2}$-shaped pulse. The oscillation wavelengths were switched at 1-ms intervals between $\lambda_{1}(794.8 \mathrm{~nm})$ and $\lambda_{2}(801.5 \mathrm{~nm})$, corresponding respectively to signal frequencies of $\mathrm{rf}_{1}=113.00 \mathrm{MHz}$ and $\mathrm{rf}_{2}=112.00 \mathrm{MHz}$ fed into the AOTF. The trigger pulses had an interval of $1 \mathrm{~ms}$ and a width of $30 \mu \mathrm{s}$. The wavelength switching timing was set to the rising edge of the trigger pulses. Pulse trains were detected by a high-speed photodetector (SiR 5, Thorlabs Inc.) and a digital oscilloscope (Waverunner 6050, Lecroy Corp.). They are shown with scales of $500 \mu \mathrm{s} / \mathrm{div}$ (Fig. 3(a)) and $5 \mu \mathrm{s} / \mathrm{div}$ (Fig. 3(b) and (c)). As Fig. 3(a) shows, switching of the oscillation wavelength was smooth except that a giant pulse was generated at the wavelength switching point. Figure 3(b) shows the oscilloscope trace of the rf signal applied to the AOTF and Fig. 3(c) shows the pulse train with a scale of 5 $\mu \mathrm{s} /$ div. These figures reveal that the relaxation oscillation occurred at the oscillation wavelength switching point. This oscillation stopped after $23 \mu$ s of triggering. Based on this time, the acoustic wave propagating in the AOTF that corresponds to $\mathrm{rf}_{1}$ was replaced with another acoustic wave that corresponds to $\mathrm{rf}_{2}$. The circuit delay of the AOTF driver was also included in the delay of $23 \mu$ s. The oscillation wavelength was changed to $\lambda_{2}$ at $3.1 \mu$ s after the $\lambda_{1}$ oscillation. The transition to the mode-locking oscillation with $\lambda_{2}$ commenced $12 \mu$ s after the first mode-locked pulse train. We observed spikes similar to those observed for the
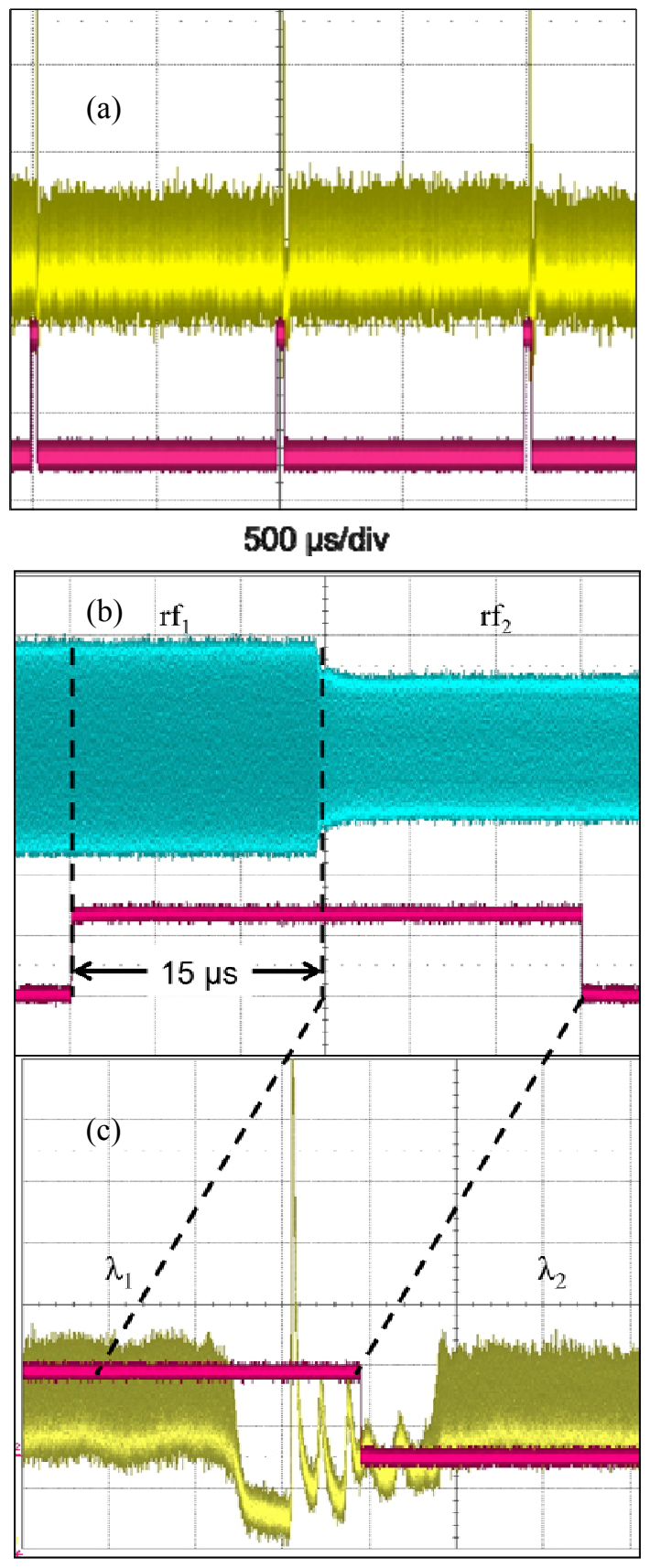

$5 \mu \mathrm{s} / \mathrm{div}$

Fig. 3 Pulse trains when the laser oscillation wavelength was switched: (a) pulse trains measured at $500 \mu \mathrm{s} / \mathrm{div}$; (b) rf signal and (c) pulse trains measured at $5.00 \mu \mathrm{s} / \mathrm{div}$. 
cw relaxation oscillation at a gap of $12 \mu \mathrm{s}$. We found that a similar $\mathrm{cw}$ relaxation oscillation occurred in the same laser system, which was determined by calculating the relaxation oscillation of a similar laser system [17]. This laser generated stable picosecond pulses due to the beat pulses generated in a frequency-shifted feedback laser [18-21]. The beat pulses induced the Kerr-lens effect in the $\mathrm{Ti}: \mathrm{Al}_{2} \mathrm{O}_{3}$ crystal. Several frequency components of the pulses agree with the longitudinal modes in the cavity. Mode-locked pulses were formed when the pumping intensity was increased due to the Kerr-lens effect. The cavity length did not affect the stability of mode-locked operation. These results demonstrate that mode locking with an AOTF is very stable. Thus, this technique is useful for MOPA systems.

\section{Conclusion}

We generated stable picosecond pulses from an electronically wavelength-tuned laser with an AOTF in its cavity. We experimentally observed a build up time within 12 $\mu$ s after the oscillations had stopped. Picosecond pulses generation from the laser was stable even when the oscillation wavelength was changed at 1-ms intervals. This modelocking method is more stable and practical for generating picosecond pulses, and it will be important in developing more advanced laser processing systems.

\section{References}

[1] P. L. Liu, R. Yen, and N. Bloembergen: Appl. Phys. Lett. 34 (1979) 864. (Journals)

[2] H. Zhang, S. M. Eaton, J. Li, A. H. Nejadmalayeri, and P. R. Herman: Opt. Exp. 15 (2007) 4182. (Journals)

[3] P. Engelhart, S. Haermann, T. Neuberg, H. Plagwitx, R. Grischke, R. Meyer, U. Klug, A. Schoonderberek, U. Stute, and R. Brendel: Prog. Photovol. 15 (2007) 521. (Journals)

[4] M. Ehrardt, G. Raclukaltis, P. Gecys, and K. Zimmer: Appl. Surf. Sci. 256 (2010) 7222. (Journals)

[5] B. Luther- Dacies, A. P. Radlinski, and A. Calka: J. Appl. Phys. 66 (1989) 3293. (Journals)

[6] Z. Kuang, W. Perrie, D. Liu, S. Edwardson, J. Vheng, G. Dearden, and K. Watkins: Appl. Surf. Sci. 255 (2009) 9040. (Journals)

[7] W. Koechner: Solid-State Laser Engineering, 6th Ed., (Springer, New York, 2006) p156. (Books)

[8] W. Koechner: Solid-State Laser Engineering, 6th Ed., (Springer, New York, 2006) p527. (Books)

[9] S. Wada, K. Akagawa, and H. Tashiro: Opt. Lett. 21 (1996) 731. (Journals)

[10] J. Geng, S. Wada, Y. Urata, and H. Tashiro: Opt. Lett. 24 (1999) 676. (Journals)

[11] Y. Maeda, M. Yumoto, N. Saito, T. Ogawa, K. Kurokawa, A. Nakano, M. Yamashita, and S. Wada: Opt. Rev. 17 (2010) 305. (Journals)

[12] L. C. Chang: Appl. Phys. Lett. 25 (1974) 323.

[13] L. C. Chang: Appl. Phys. Lett. 25 (1974) 370. (Journals)

[14] L. C. Chang: Proc. SPIE 131 (1978) 2. (Journals)

[15] L. C. Chang: Opt. Eng. 20 (1981) 824. (Journals)

[16] M.Yumoto, Y. Maeda, N. Satio, T. Ogawa, M. Yamashita, and S. Wada: J. Jpn. Appl. Phys. 47 (2008) 8411. (Journals)
[17] N. Saito, S. Wada, and H. Tashiro: J. Opt. Soc. Am. B 18 (2001) 1288. (Journals)

[18] F. V. Kowalski, J. A. Squier, and J. T. Pinckney: Appl. Phys. Lett. 50 (1987) 711. (Journals)

[19] F. V. Kowalski, S. J. Shattil, and P. D. Hale: Appl. Phys. Lett. 53 (1988) 734. (Journals)

[20] G. Bonnet, S. Balle, Th. Kraft, and K. Bergmann: Opt. Commun. 123 (1996) 790. (Journals)

[21]Q. Wu, J. Y. Zhou, X. G. Huang, Z. X. Li, and Q. X. Li: J. Opt. Soc. Am. B10 (1993) 2080. (Journals)

(Received: November 26, 2010, Accepted: December 24, 2010) 